\title{
Cytochrome 4ZI Expression is Associated with Poor Prognosis in Colon Cancer Patients
}

\author{
Yousef M Al-saraireh (ID) \\ Fatemah OFO Alshammari $^{2}$ \\ Ahmed MM youssef (D) $^{3}$ \\ Sameeh Al-Sarayreh ${ }^{4}$ \\ Ghadeer H Almuhaisen ${ }^{5}$ \\ Nedal Alnawaiseh (D) $^{6}$ \\ Jehad M Al-Shuneigat ${ }^{4}$ \\ Hamzeh M Alrawashdeh ${ }^{7}$ \\ 'Department of Pharmacology, Faculty of \\ Medicine, Mutah University, Al-Karak, \\ Jordan; ${ }^{2}$ Department of Medical \\ Laboratory Technology, Faculty of Health \\ Sciences, The Public Authority for \\ Applied Education and Training, \\ Shuwaikh, Kuwait; ${ }^{3}$ Department of \\ Pharmacology, Faculty of Pharmacy, \\ Mutah University, Al-Karak, Jordan; \\ ${ }^{4}$ Department of Biochemistry and \\ Molecular Biology, Faculty of Medicine, \\ Mutah University, Al-Karak, Jordan; \\ ${ }^{5}$ Department of Microbiology and \\ Pathology, Faculty of Medicine, Mutah \\ University, Al- Karak, Jordan; \\ ${ }^{6}$ Department of Public Health, Faculty of \\ Medicine, Mutah University, Al-Karak, \\ Jordan; ${ }^{7}$ Department of Ophthalmology, \\ Sharif Eye Centers, Irbid, Jordan
}

Correspondence: Yousef M Al-saraireh Department of Pharmacology, Faculty of Medicine, Mutah University, PO. Box: 7 , Al-Karak, 61710, Jordan

Tel +962799172658

Email Yousef.sar@mutah.edu.jo
Purpose: Colon cancer is a leading cause of mortality worldwide. It has a relatively poor prognosis; therefore, new therapies are needed. One of the tumour-related enzymes that has gained considerable interest is CYP4Z1. This enzyme has been expressed in many tumours and has been hypothesized as a potential biomarker or target for novel anticancer therapies. Patients and Methods: CYP4Z1 overexpression was immunohistochemically examined in a large panel of colon tissue types including normal, benign, primary and metastatic ones, and the enzyme's relation to histopathological features and patient survival was evaluated.

Results: A high CYP4Z1 expression was observed in benign, primary and metastatic colon tissues compared to a weak or lack of expression in normal tissues. Importantly, there was a significant differential in CYP4Z1 expression where it was stronger in metastatic, primary and benign, respectively $(p<0.05)$. A significantly high rate of CYP4Z1 expression was found in high histological grades and late stages of the disease, where its expression was more evident in patients with metastasis in the lymph nodes $(p<0.05)$. Interestingly, CYP4Z1 expression was identified an independent prognostic predictor of poor overall survival of colon cancer patients $(p=0.003)$.

Conclusion: CYP4Z1 was distinctly overexpressed in benign, primary and metastatic colon tissues compared to corresponding normal tissues. This differential in CYP4Z1 expression across different types of colon tissues strongly supports CYP4Z1 as potential biomarker and target for novel anticancer therapy development.

Keywords: cancer, colon cancer, cytochrome P450, cytochrome 4Z1, immunohistochemistry

\section{Introduction}

Colon cancer is the second leading cause of cancer mortality and third in terms of morbidity worldwide. ${ }^{1}$ According to the World Health Organization (WHO), the colon cancer burden rose to 1.8 million new cases and about 862,000 deaths from colon cancers globally in 2018. In the Middle East, there are no accurate statistics for the colon cancer burden. However, the WHO revealed that the mortality and morbidity of colon cancer are on the rise, especially in developing countries. $^{2}$ This is mainly attributed to poor screening programs and ineffective management of risk factors and treatment regimens. ${ }^{3}$ Recently, many new therapies for colon cancer have been developed, including laparoscopic surgery; resection of metastatic disease; radiotherapy for some forms of metastatic disease; adjuvant and neo-adjuvant therapies, and, finally, palliative chemotherapy. However, the cure and relative survival rates for colon cancer patients drop in metastatic situations. ${ }^{4,5}$ Therefore, it is necessary to develop effective new therapies for colon cancer. 
Specific cytochrome P450 (CYP450) individual forms were shown to be selectively expressed in both normal and cancer tissues in humans. ${ }^{6-8}$ One enzyme that has attracted particular interest is CYP4Z1. It is an extrahepatic enzyme that is considered as an orphan form due to its less defined substrate recognition and physiological activities. ${ }^{9}$ This novel enzyme was selectively expressed in healthy breast tissues with an absence of expression in other healthy normal human tissues. ${ }^{10}$ Interestingly, a unique strong CYP4Z1 expression was identified in tumours of the breast, ovary, prostate, bladder and cervix compared to normal healthy tissues. ${ }^{6,7,11-15}$ This overexpression was correlated with an increasing grade of tumour and poor patient outcomes. ${ }^{11,12,15,16}$ Moreover, CYP4Z1 was aberrantly expressed at the cell membrane of breast cancer cells and promoted the production of antiCYP4Z1autoantibodies in the patients' sera of breast cancer, as opposed to none of the healthy controls. ${ }^{17}$ This expression has promoted tumour growth, angiogenesis and the spread of tumour cells. ${ }^{18}$ Furthermore, treatment of breast cancer cells, glucocorticoids and progesterone led to conditional regulation of CYP4Z1 expression in these cells. However, this expression was decreased upon treatment with the steroid receptor blocker, mifepristone. ${ }^{19}$

In an attempt to identify the catalytic properties of CYP4Z1, several functional studies investigated the biochemical mechanisms by which the CYP4Z1 acts. CYP4Z1 catalyzed the $\omega$-hydroxylation of lauric acid and myristic acid, generating different monohydroxylated metabolites at several positions. ${ }^{20}$ Additionally, CYP4Z1 was able to metabolize arachidonic acid to 20hydroxyeicosatetraenoic acid (20-HETE), resulting in the promotion of tumour angiogenesis and tumour development. ${ }^{18}$ However, a recent study utilizing the CYP4Z1 recombinant enzyme system demonstrated the lack of 20-HETE metabolites in the arachidonic acid reaction with CYP4Z1. The study further proposed that CYP4Z1 may enhance breast tumorigenesis without direct synthesis of 20-HETE. ${ }^{21}$

In a search for specific substrates for CYP4Z1, a limited number of studies examined the substratebinding mode of this enzyme. Using recombinant CYP4Z1 mutants and the homology model of CYP4Z1, Arg487, Asn381, Ser383, Ser222, Ser113, and Asn381 were identified as key residues for substrate binding of CYP4Z1. ${ }^{22,23}$ Moreover, the screening of different luminogenic substrates with CYP4Z1-containing enzyme bags led to the identification of luciferin benzyl ether as the best substrate. ${ }^{23}$ Recently, a first selective mechanism-based inhibitor called 8-[(1H-benzotriazol-1-yl $) \neg$ amino $] \neg$ octanoic acid for CYP4Z1 was developed and exhibited a potent inhibitory concentration for CYP4Z1 and low inhibition effect for other CYP enzymes. ${ }^{24}$

In a recent small-scale study, ${ }^{13}$ we identified CYP4Z1 to be highly expressed in colon cancers. On this basis, we have extended our observations to characterize aberrant CYP4Z1 expression in a large scale of colon tissue types, including normal, benign, primary and metastatic, and to investigate its relation to baseline demographic, clinicopathologic and survival features.

\section{Patients and Methods}

\section{Tissue Specimens}

A panel of formalin-fixed, paraffin-embedded (FFPE) specimens were collected from the Pathology Department, King Hussein Medical Hospital, and King Abdullah University Hospital, Jordan. This panel consisted of 60 colon adenocarcinomas, ten colon tubular adenomas, 69 metastatic adenocarcinomas from the colon (61 lymph node and eight liver metastasis), and 30 normal colon tissues. Patients who received preoperative chemotherapy or radiotherapy were excluded from the study. Data regarding age, tumour histopathology, histological grade, and stage of the disease were extracted from the patients' files. All patients' data were kept confidential and anonymous. The use of human tissue specimens in this study was approved by the Institutional Review and Ethics Committee, Faculty of Medicine, University of Mutah (3012021). Moreover, an exemption of written informed consent for the utilization of FFPE colon tissue samples was granted from the above mentioned committee. The study complies with regulations set by Declaration of Helsinki (2013).

\section{Immunohistochemistry}

In this study, a polyclonal antibody raised in rabbits against the internal region of the amino acid peptide (40-120) of the human CYP4Z1 protein was used to identify sites of CYP4Z1 immunoreactivity (NBP1-91817, Novus biological, USA). Antibody specificity towards CYP4Z1 protein was checked by Western blotting using CYP4Z1 isogenic cell lysates (data not shown). Moreover, the specificity of primary antibody was also confirmed by pre-incubating CYP4Z1 antibody with CYP4Z1 blocking peptide (H00199974-P01, Novus biological, USA) for one hour at 
room temperature. The mixture was then utilized in place of CYP4Z1 primary antibody to inhibit subsequent primary antibody binding to CYP4Z1 epitope in sample. The staining intensity was compared between the samples incubated with CYP4Z1 antibody and blocked antibody. Immunohistochemical detection of CYP4Z1 was performed using a peroxidase polymer-based detection system.

Sections of different colon tissue types were deparaffinized in xylene and rehydrated to cold water through serial dilutions of alcohol. To block endogenous peroxidase activity, tissues were treated with $3 \%$ hydrogen peroxide at room temperature for ten minutes. Tissues then underwent a heat-based antigen retrieval process in a 10 $\mathrm{mM}$ citrate buffer, $\mathrm{pH} 6.0$, for 20 minutes. The nonspecific antibody binding was blocked with $2.5 \%$ normal goat serum for 20 minutes in a humidified chamber. The primary antibody was applied at a concentration of $5 \mu \mathrm{g} /$ $\mathrm{mL}$ overnight at $4^{\circ} \mathrm{C}$. Sites of CYP4Z1 immunoreactivity were identified using ImmPRESS (Peroxidase) Polymer goat Anti-Rabbit IgG Reagent for 30 minutes at room temperature (MP-7451, Vector Laboratories, Burlingame, USA). To visualize immunoreactivity, sections were incubated with diaminobenzidine chromogen solution as a substrate (Vector Laboratories Ltd, Peterborough, UK). To establish the presence and absence of CYP4Z1 immunoreactivity and its cellular localization, a haematoxylin solution was used to counterstain tissue sections. Tissue sections of breast cancer that had previously been shown to express CYP4Z1 by immunohistochemistry were utilized as a positive control. ${ }^{13}$ As negative control samples, colon tissue sections were incubated with normal goat serum instead of a primary antibody. The resulting slides were examined using a Leica DMRB microscope (Leica DMRB, Wetzlar, Germany) and images were digitally captured using AcQuis Biosystem (Synoptics, Cambridge, UK).

\section{Scoring}

Stained sections were semi-quantitatively and independently examined by two pathologists. Cells showing yellow or brown membranous or cytoplasmic immunostaining were considered positive. Tissue specimens were scored for the density and extent of CYP4Z1 expression on the following scale: negative (0), weak (1), intermediate (2) and high (3). The score "negative" indicated a lack of expression or if less than $5 \%$ of tumour cells showed positive immunostaining. A score of "weak" indicated cells having an expression lower than $33 \%$. If cells showed an expression of 34-66\%, a score of "intermediate" was used. When the cells had an expression higher than $67 \%$, a score of "high" was applied.

\section{Statistical Analysis}

SPSS software version 19 (Statistical Packages for Social Sciences) was used to analyse the statistical data. All values were expressed in simple measures of frequency and percentage. Pearson's chi-square test, with the application of an ANOVA test, was used to assess the difference between the discrete variables. Kaplan-Meier method was used to calculate the overall survival of patients and statistical significance was assessed by Log rank test. Cox regression was used to calculate the hazard ratio (HR) and 95\% confidence limits $(\mathrm{Cl})$. A $P$ value of less than 0.05 was considered statistically significant.

\section{Results}

\section{Baseline Demographic and Clinicopathologic Features for Colon Cancer Patients}

The baseline demographic and clinicopathologic features of the 169 patients are listed in Table 1 . The current study consisted of 110 males and 59 females. The mean age for patients was estimated at $54.1 \pm 14.3$ years. Most of the normal samples were normal colon tissue adjacent to tumours (11.8\%, 20 cases) and the remaining were normal healthy tissues (5.9\%, ten cases). Moreover, all the benign colorectal tumours were tubular adenomas $(5.9 \%$, ten cases). The primary colorectal tumours consisted of 60 (35.5\%) adenocarcinomas, where $8.3 \%$ (five cases) of them were mucinous adenocarcinomas. Of the 69 metastatic tumours, most were lymph node metastasis comprising $36.1 \%$ (61 cases) of all patients and only 4.7\% (eight cases) of metastatic tumours were from liver metastasis. The majority of colorectal cancer patients were grade II (31.4\%, 53 cases) and III (34.3\%, 58 cases), while grade I constituted only $10.7 \%$ (18 cases). Of 60 patients with primary colorectal cancer, most were stage II $(55 \%, 33$ cases) and III (30\%, 18 cases), while other stages were I ( $10 \%$, six cases) and IV (5\%, three cases). Moreover, the majority of the cases involved had tumour depth of invasion of T4 $(36,60 \%)$, whilst other cases had tumour depth of invasion of T1 $(1.7 \%$, one case), T2 (8.3\%, five case) and T3 (30\%, 18 cases). Additionally, 60\% (36 cases) of the primary tumours had no lymph node metastasis (N0), whereas $25 \%$ (15 cases) had metastasis to three regional 
Table I Baseline Demographic and Clinicopathologic Features

\begin{tabular}{|c|c|c|c|c|c|}
\hline \multirow[b]{2}{*}{ Characteristics } & \multicolumn{4}{|c|}{ Cytochrome 4ZI Expression } & \multirow[b]{2}{*}{$P$ value } \\
\hline & Negative & Weak & Intermediate & High & \\
\hline \multicolumn{6}{|l|}{ Gender } \\
\hline Male $(n=\mid 10,65.1 \%)$ & $40(36.4 \%)$ & $26(23.6 \%)$ & $13(1 \mid .8 \%)$ & 31 (28.2\%) & 0.373 \\
\hline Female $(n=59,34.9 \%)$ & $23(39 \%)$ & 9 (I5.3\%) & $8(13.6 \%)$ & $19(32.2 \%)$ & \\
\hline \multicolumn{6}{|l|}{ Age: } \\
\hline$<60(n=53,31.4 \%)$ & $48(41.4 \%)$ & $26(22.4 \%)$ & $14(12.1 \%)$ & $28(24.1 \%)$ & 0.011 \\
\hline$\geq 60(n=53,31.4 \%)$ & 15 (28.3\%) & $9(17 \%)$ & 7 (I3.2\%) & $22(4 I .5 \%)$ & \\
\hline \multicolumn{6}{|l|}{ Histological type } \\
\hline Normal $(n=30,17.8 \%)$ & $15(50.0 \%)$ & II (36.7\%) & $2(6.7 \%)$ & $2(6.7 \%)$ & 0.028 \\
\hline Benign $(n=10,5.9 \%)$ & $3(30 \%)$ & $3(30 \%)$ & I (I0\%) & $3(30 \%)$ & \\
\hline Primary $(n=60,35.5 \%)$ & $19(31.7 \%)$ & $12(20 \%)$ & II (I8.3\%) & $18(30 \%)$ & \\
\hline Metastasis $(n=69,40.8 \%)$ & $26(37.7 \%)$ & $9(13 \%)$ & 7 (10.1\%) & 27 (39.1\%) & \\
\hline \multicolumn{6}{|l|}{ Histological grade } \\
\hline I $(n=18,10.7 \%)$ & $3(16.7 \%)$ & 4 (22.2\%) & $2(11.1 \%)$ & $9(50 \%)$ & 0.029 \\
\hline II $(n=53,31.4 \%)$ & $13(24.5 \%)$ & $10(18.9 \%)$ & $9(17 \%)$ & $21(39.6 \%)$ & \\
\hline III $(n=58,34.3 \%)$ & $29(50 \%)$ & 7 (12.1\%) & $7(12.1 \%)$ & $15(25.9 \%)$ & \\
\hline \multicolumn{6}{|l|}{ Histological stage } \\
\hline$I(n=6,10.0 \%)$ & $2(33.3 \%)$ & I (I6.7\%) & I (I6.7\%) & $2(33.3 \%)$ & 0.035 \\
\hline II $(n=33,55.0 \%)$ & 9 (27.3\%) & 7 (21.2\%) & $6(18.2 \%)$ & II (33.3\%) & \\
\hline III $(n=18,30.0 \%)$ & 7 (38.9\%) & $4(22.2 \%)$ & $4(22.2 \%)$ & $3(16.7 \%)$ & \\
\hline IV $(n=3,5.0 \%)$ & I (33.3\%) & $0(0.0 \%)$ & $0(0.0 \%)$ & $2(66.7 \%)$ & \\
\hline \multicolumn{6}{|l|}{ Tumour invasion } \\
\hline TI+T2 (n=6, $10 \%)$ & $2(33.3 \%)$ & I (16.7\%) & I (I6.7\%) & $2(33.3 \%)$ & 0.545 \\
\hline$T 3+T 4(n=54,90 \%)$ & 17 (3I.5\%) & II (20.4\%) & $10(18.5 \%)$ & $16(29.6 \%)$ & \\
\hline \multicolumn{6}{|l|}{ Lymph node metastasis } \\
\hline No $(n=36,60 \%)$ & 8 (22.2\%) & $8(22.2 \%)$ & 7 (19.4\%) & $13(36.1 \%)$ & 0.030 \\
\hline $\mathrm{NI}(\mathrm{n}=15,25 \%)$ & 8 (53.3\%) & $2(13.3 \%)$ & $3(20 \%)$ & $2(13.3 \%)$ & \\
\hline N2 (n=9, 15\%) & $3(33.3 \%)$ & $2(22.2 \%)$ & I (II.I\%) & $3(33.3 \%)$ & \\
\hline \multicolumn{6}{|l|}{ Distant metastasis } \\
\hline M0 $(n=56,93.3 \%)$ & $18(32.1 \%)$ & $12(2 \mid .4 \%)$ & $10(17.9 \%)$ & $16(28.6 \%)$ & 0.373 \\
\hline MI $(n=4,6.7 \%)$ & I (25\%) & $0(0.0 \%)$ & I (25\%) & $2(50 \%)$ & \\
\hline
\end{tabular}

lymph nodes and 15\% (nine cases) had metastases to six regional lymph nodes. Furthermore, distant metastasis only occurred in $6.7 \%$ of patients (four cases), while the majority of patients had no distant metastasis $(93.3 \%, 56$ cases).

\section{Prevalence of CYP4ZI Expression in Normal Colon and Benign Colorectal}

\section{Cancers}

The scoring criteria for CYP4Z1 expression and immunostaining pattern are shown in Figure 1. CYP4Z1 immunoreactivity was predominantly localized to the membrane or cytoplasm of cells without any considerable staining in nuclei. Of the $50 \%$ positive normal cases (15 out of 30 ), expression was low in 11 cases $(73.3 \%)$ where it was heterogeneous and varied in intensity from one case to another and even from one area to another in the same tissue (Figure 2) (Table 1). Most positive cases were normal colon tissues adjacent to tumours (70\%) and the remaining were normal healthy tissues (30\%). Importantly, CYP4Z1 expression was confirmed by using appropriate positive and negative controls and by inhibition of immunostaining using CYP4Z1protein as a blocking step. The positive control breast cancer tissue exhibited high CYP4Z1 expression, while negative control displayed no expression at all. There was weak to no CYP4Z1 

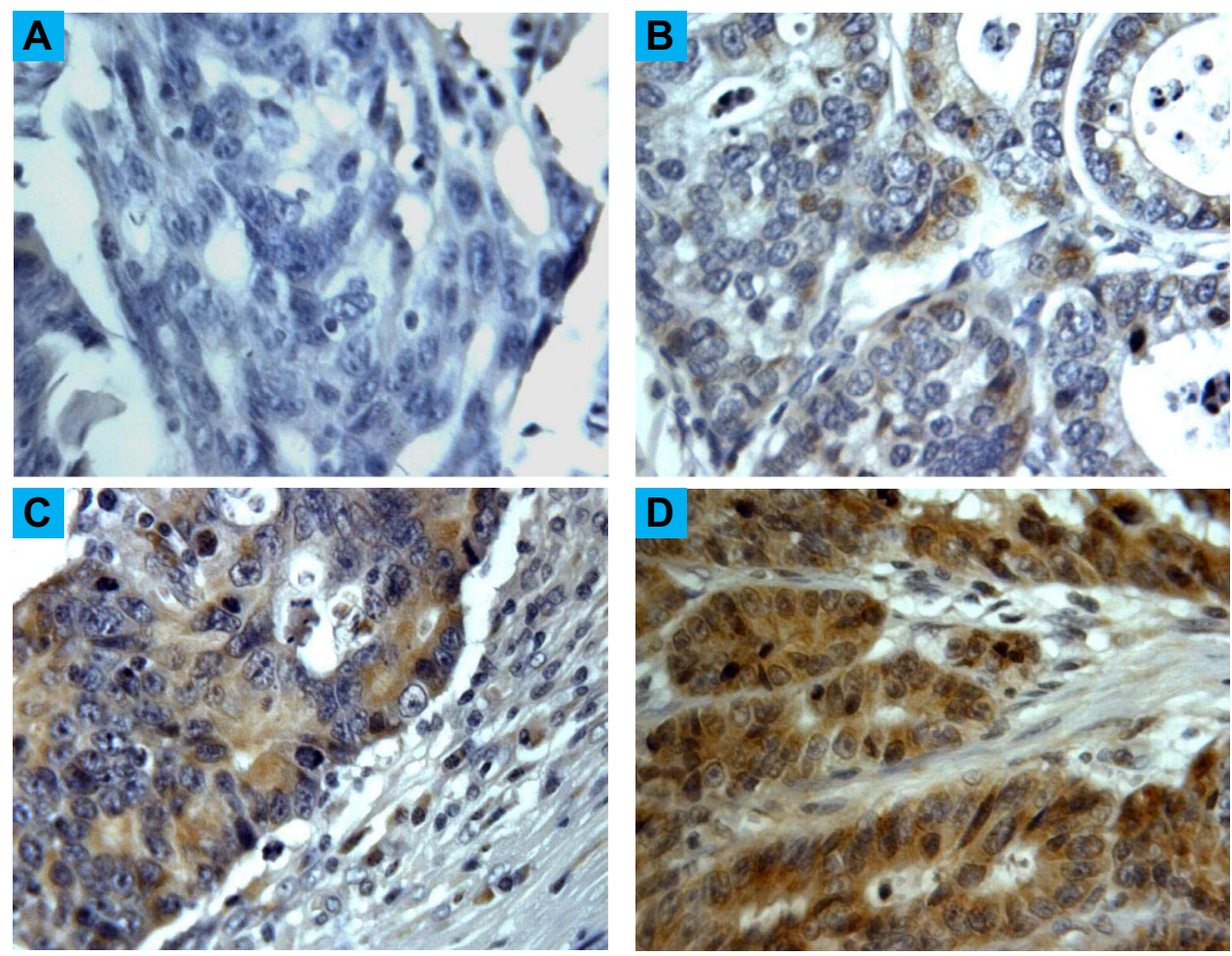

Figure I Different scores and immunostaining pattern of CYP4ZI expression in colon cancers. CYP4ZI immunoreactivity was predominantly localized to the membrane or cytoplasm of cells without any considerable staining in nuclei. (A) Score "negative" showing no expression in the tissue at all. (B) Score "low" showing expression less than $33 \%$ of cells, (C) score "moderate" showing expression in 34-66\% of the cells, (D) score "strong" showing expression in more than $67 \%$ of the cells. Magnification (X400).

expression displayed in colon and breast cancer tissues incubated with mixture of primary antibody and blocking peptide (Figure 3 ).

Most benign tumours (seven cases, 70\%) showed more intense CYP4Z1 immunoreactivity than those seen in the normal tissues. Of seven positive cases, three cases (30\%) showed high expression, whereas others demonstrated low expression in three cases $(30 \%)$ and intermediate expression in one case $(10 \%)$. Staining was characterized as heterogonous and some areas showed completely negative expression. The comparison between the expression of CYP4Z1 in normal tissues and benign colorectal tumour tissues showed a borderline significant association $(P=$ 0.075) (Figure 4).

\section{Prevalence of CYP4ZI Expression in Primary Colorectal Cancers}

CYP4Z1 immunoreactivity was recognized in the majority of primary colorectal tumours (41 cases, 68.3\%). In a high percentage of the colorectal cancers, staining was high in 18 cases $(30.0 \%)$, whereas others exhibited intermediate expression in 11 cases $(18.3 \%)$ and low expression in 12 cases (20.0\%). Moreover, no apparent intratumour heterogeneity was observed across all tissue sections examined (Figure 2).

The relationships between the expression of CYP4Z1 and different demographic and clinicopathologic features were investigated. There was no significant relation between the expression of CYP4Z1 and patients' gender, the tumour depth of invasion, and distant metastasis. CYP4Z1 was expressed in 63.6\% (70 cases) of males and $61.1 \%$ (36 cases) of females. Moreover, a high frequency of CYP4Z1 expression was found in tumours with a tumour depth of invasion, particularly in T3 and T4 tumours, but not in $\mathrm{T} 1$ and $\mathrm{T} 2$ tumours, although this finding did not reach statistical significance. The same tendency of CYP4Z1 expression was seen in distant metastasis. However, CYP4Z1 expression was strongly associated with tumour histological grade, histological stage, lymph node metastasis, and the pathology type of the tumour $(p$ value $<0.05$ ). The different grades of colorectal cancers all exhibited an elevated level of CYP4Z1 expression: grade 1 (50\%, nine cases), grade $2(39.6 \%, 21$ cases) and grade $3(25.9 \%, 15$ cases). In addition, a significantly higher rate of CYP4Z1 expression was 

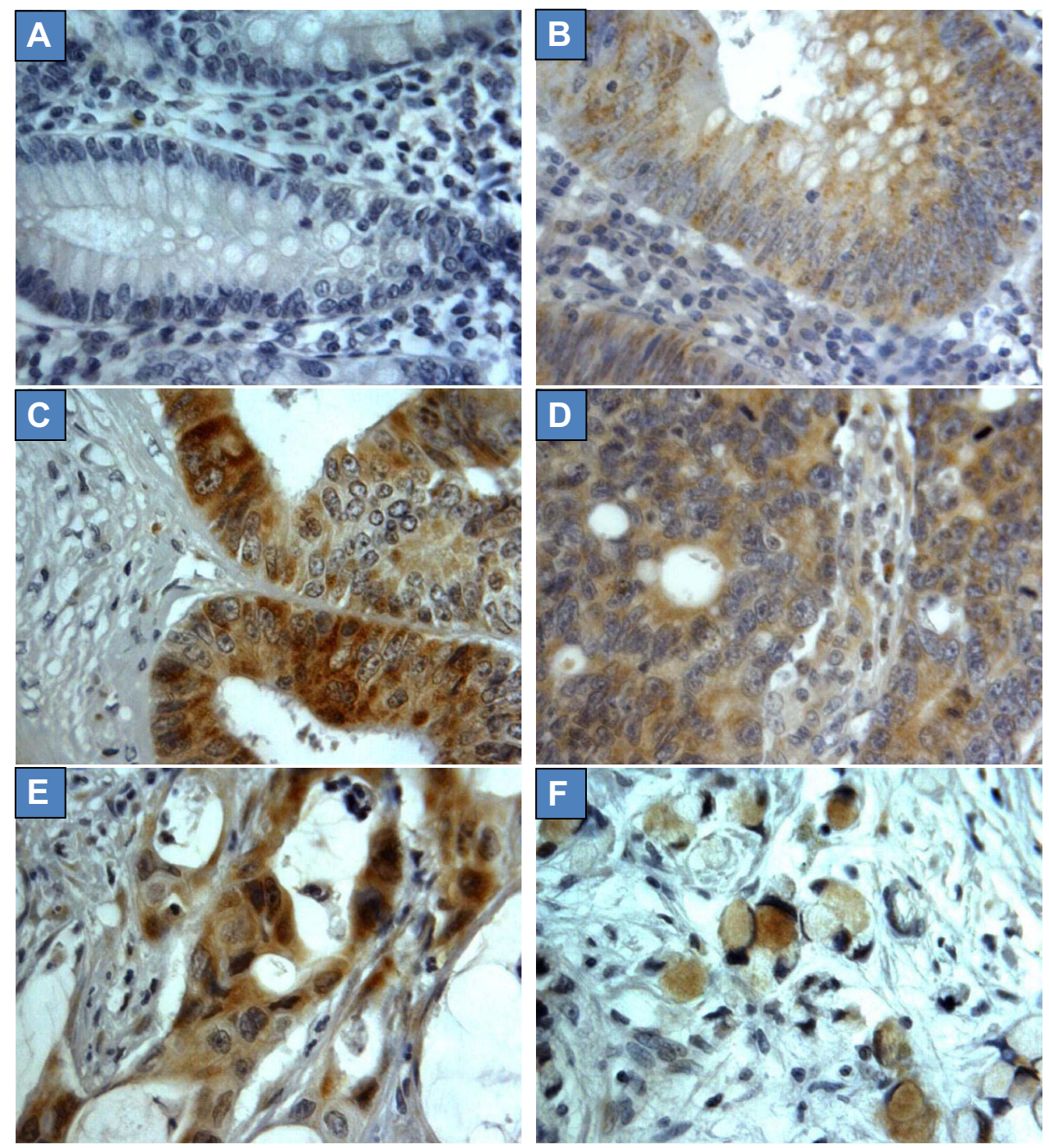

Figure 2 CYP4ZI expression in different types of colon tissues. Tumours were classified on the basis of histological type. (A) Normal colon tissue, (B) colon tubular adenoma, (C) colon adenocarcinoma, (D) metastatic adenocarcinoma from colon, (E) Metastatic mucinous adenocarcinoma and (F) metastatic signet-ring cell carcinoma. Magnification (X400).

observed in the advanced stage of the disease compared with the early stages of the disease. Moreover, lymph node metastases were more frequently found in colon cancer with CYP4Z1 expression as opposed to normal samples. Importantly, CYP4Z1 was differentially expressed between normal tissues and primary colorectal tumours $(P=0.014)$. Moreover, there was a differential in CYP4Z1 expression between benign and primary tumours, although this finding showed a borderline significant correlation $(P=0.065)$ (Figure 4).

\section{Prevalence of CYP4ZI Expression in Metastatic Colorectal Cancers}

CYP4Z1 expression was identified in the majority $62.3 \%$ (43 cases) of metastatic samples (Figure 2). Of 43 positive metastatic cases, CYP4Z1 expression was high in 39.1\% (27) cases, while others showed intermediate expression in $10.1 \%$ (seven cases) and low expression in 13\% (nine) cases. The comparison between CYP4Z1 expression in metastatic deposits and normal and benign tissues showed a significant association ( $P=0.005$ and $P=0.024$, respectively). However, there was no significant difference in CYP4Z1 expression between primary colorectal cancers and metastatic deposits $(P=0.725)$ (Figure 4).

\section{Correlation of CYP4ZI Expression with Colon Cancer Patients' Survival}

The survival of 60 colon cancer patients was followed for 130 months. To aid statistical comparison, CYP4Z1 expression scores were grouped as negative expression 

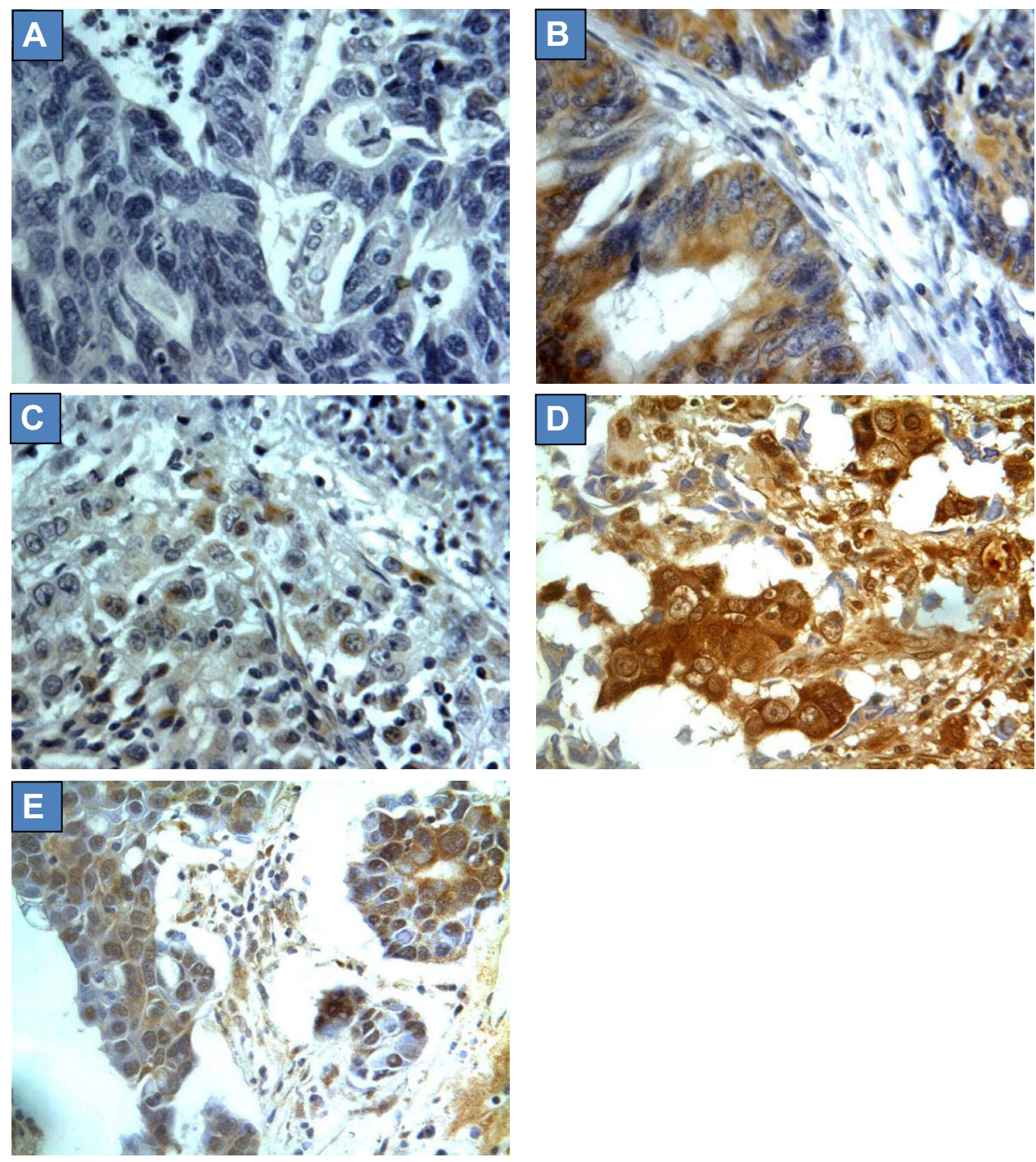

Figure 3 CYP4ZI expression in different types of experimental controls. (A) No CYP4ZI expression in colon cancer tissue incubated with normal goat serum instead of CYP4ZI primary antibody (negative control), (B) high CYP4ZI expression was displayed in colon cancer tissue incubated with CYP4ZI primary antibody, (C) weak to no expression of CYP4ZI was exhibited in colon cancer tissue incubated with mixture of primary antibody and blocking peptide, (D) high CYP4ZI expression was displayed in breast cancer tissue incubated with CYP4ZI primary antibody (positive control) and (E) very weak expression of CYP4ZI was observed in breast cancer tissue incubated with mixture of primary antibody and blocking peptide. Magnification (x400).

(negative, 19 patients) and positive expression (weak, intermediate and high, 41 patients). Kaplan-Meier curve and $\log$ rank test were used to evaluate patient survival data with respect to CYP4Z1 expression groups. Survival analysis revealed that patients characterized with CYP4Z1 expression had poor overall survival (43.9\%) compared to those with negative CYP4Z1 expression (63.2\%) ( $\mathrm{p}=$ 0.041) (Figure 5). The median overall survival for patients characterized with CYP4Z1 expression was $96 \pm 5$ months compared to $116 \pm 4$ months for patients with negative CYP4Z1 expression. By using univariate Cox regression analysis, only CYP4Z1 expression was significantly correlated with survival of colon cancer patients with relative hazard ratio of 1.025 ( $P=0.001,95 \%$ CI: $1.013-1.073)$. Furthermore, the multivariate Cox regression analysis revealed similar results to that obtained by univariate Cox regression analysis and identified CYP4Z1 expression as an independent prognostic factor of poor overall survival of colon cancer patients $(P=0.003$; HR $1.038,95 \%$ CI: 1.013-1.154) (Table 2).

\section{Discussion}

The present study has investigated the presence of CYP4Z1 expression in relation to colon tissue types including normal, benign, primary and metastatic. In a study utilizing a small number of samples, we have 


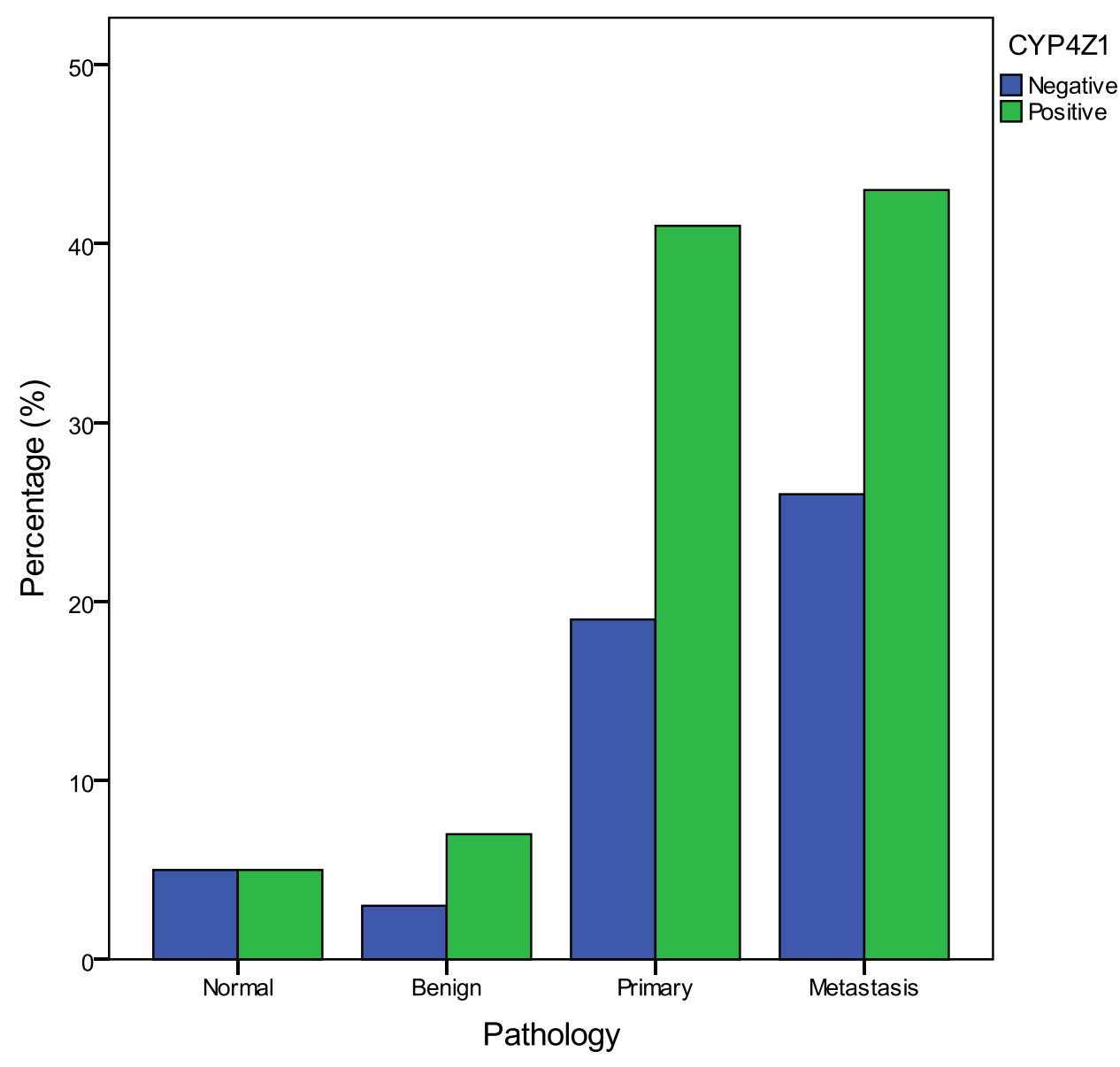

Figure 4 CYP4ZI expression in normal, benign, primary and metastatic colon samples.

shown the overexpression of CYP4Z1 in primary colon tumours and the lack of expression in corresponding normal tissues. ${ }^{13}$ The existing data support and broaden our observations in which CYP4Z1 expression was elevated in primary colon adenocarcinomas compared to low or undetectable expression in corresponding colon normal tissues. This subtype of colon cancer is the major pathological subtype found in patients suffering from this cancer. ${ }^{25}$ Moreover, benign colon tumours also showed a similar pattern of expression to primary tumours but the expression was weak and heterogeneous. However, this contrasts with previous studies investigating the expression profile of individual forms of CYP450, including CYP4Z1 in colon cancers. These studies indicated weak or absent CYP4Z1 expression in colon cancers. ${ }^{26,27}$

Several reports demonstrated the expression of CYP4Z1 in a wide variety of primary tumours such as breast, ovary, prostate, bladder and cervix and a lack of expression in normal tissues. ${ }^{6,10-13,15,16,18,28}$ However, there is no information on the presence of CYP4Z1 in metastatic disease. Several studies reported a lack of CYP4Z1 expression in metastatic colon cancers. ${ }^{26,27}$ Conversely, one of the key findings of the current study is the high frequency of CYP4Z1 expression at a greater intensity in the majority of metastatic deposits. Indeed, this study is the first describing CYP4Z1 overexpression in metastatic colon cancers. A similar prototype of CYP4Z1 expression was detected in metastatic ovarian cancer, although it was at a somewhat lower frequency. ${ }^{11}$

The present investigation has also focused on the differential expression of CYP4Z1 concerning colon tissue types, including normal, benign, primary and metastatic. This study is the first indicating that CYP4Z1 expression was higher in metastatic tissue compared to normal and benign tissues, as well as in primary tissue versus normal and benign tissues. Moreover, a higher CYP4Z1 expression in metastatic tissue compared to primary tissues was apparent, although this finding did not show a significant correlation. This differential in CYP4Z1 expression between colon tissue subtypes may indicate that CYP4Z1 can be associated with colon cancer progression. 


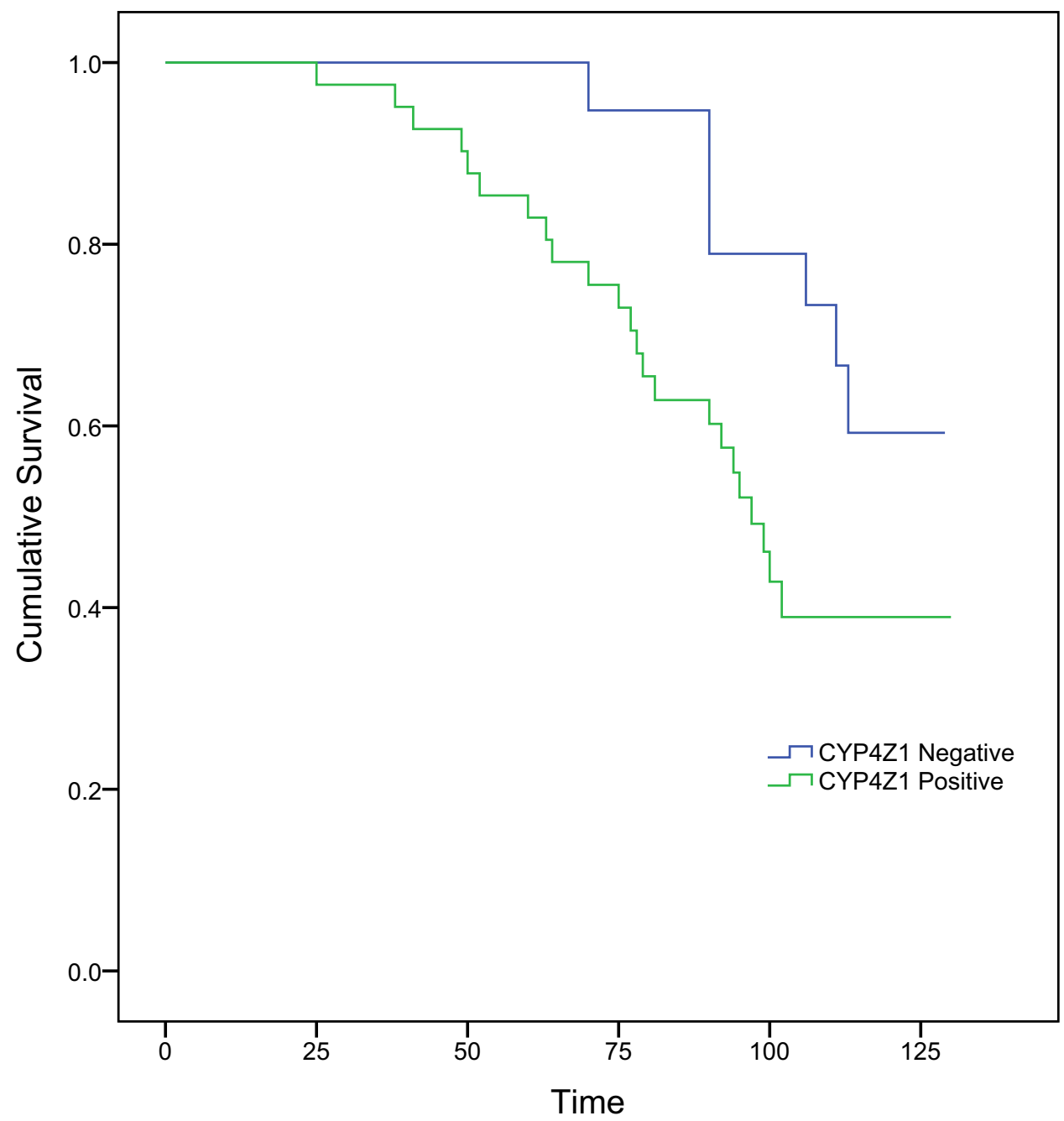

Figure 5 Kaplan-Meier curve showing overall survival of colon cancer patients.

CYP4Z1 expression has been reported to be associated with aggressive features in many cancers and is linked with poor survival, particularly in ovarian, prostate, cervical and triple negative breast cancers. ${ }^{11,12,15,16}$ In this study, CYP4Z1 expression was associated with low survival rate of colon cancer patients and connoted poor prognosis, which is a novel finding. Moreover, there was a significant association between CYP4Z1 expression and tumour histological grade, the status of lymph node metastasis, and histological stage of colon cancers. CYP4Z1

Table 2 Univariate and Multivariate Analyses of Prognostic Variables Correlated with Colon Cancer Patient Survival

\begin{tabular}{|l|c|c|c|c|c|c|}
\hline \multirow{2}{*}{ Prognostic Variable } & \multicolumn{3}{|c|}{ Univariate } & \multicolumn{3}{c|}{ Multivariate } \\
\cline { 2 - 7 } & HR & $\mathbf{9 5 \% C l}$ & P-value & HR & $95 \%$ CI & P-value \\
\hline Age & 1.003 & $0.979-1.027$ & 0.818 & 0.978 & $0.950-1.005$ & 0.111 \\
Gender & 0.986 & $0.910-1.135$ & 0.334 & 1.465 & $0.988-1.845$ & 0.543 \\
Histological grade & 0.840 & $0.529-1.334$ & 0.460 & 1.520 & $0.908-2.546$ & 0.112 \\
Histological stage & 1.399 & $0.868-2.254$ & 0.168 & 2.555 & $0.668-9.780$ & 0.171 \\
Tumour invasion & 1.073 & $0.670-1.718$ & 0.768 & 0.802 & $0.394-1.635$ & 0.544 \\
Lymph node metastasis & 1.192 & $0.720-1.975$ & 0.494 & 0.875 & $0.290-2.642$ & 0.813 \\
Distant metastasis & 1.246 & $0.294-5.273$ & 0.765 & 0.306 & $0.046-2.048$ & 0.222 \\
CYP4ZI expression & 1.025 & $1.013-1.073$ & 0.001 & 1.038 & $1.013-1.154$ & 0.003 \\
\hline
\end{tabular}


expression was gradually elevated with rising tumour histological grade. This pattern of association has been reported before where the CYP4Z1 was significantly expressed in high grades of tumours. ${ }^{6,10,13}$ Similarly, CYP4Z1 expression was positively correlated with the histological stage, where its expression was significantly more elevated in advanced stages than in the early stages of the disease. Interestingly, CYP4Z1 expression was strongly elevated in colon cancer in patients with metastasis in the lymph nodes. This tendency of CYP4Z1 expression was also observed in lymph node metastasis of breast and cervical cancer patients. ${ }^{13,15,16}$ Our findings are suggestive of the function of CYP4Z1 in colon cancer progression and metastasis.

Although this is the first study correlating CYP4Z1 with colon tissue types, there have been several reports that recognize the mechanistic function of CYP4Z1. By using cell-line models, CYP4Z1 was conditionally expressed in the cells of breast cancer upon treatment with corticosteroids. ${ }^{18,19}$ Its expression was displayed on the membrane of breast cancer cells as opposed to normal cells. ${ }^{17}$ This led to the generation of CYP4Z1 autoantibodies in breast cancer patients' sera and has been proposed as a biomarker for breast cancer malignancy. ${ }^{29}$ Moreover, CYP4Z1 expression in breast cancer has encouraged both in vitro and in vivo studies to interrogate the effect of CYP4Z1 on cell and tumour development. CYP4Z1 expression was found to promote tumour angiogenesis, proliferation and spread of tumour cells. All of these effects came with high levels of vascular endothelial growth factor-A and a reduction in the levels of the tissue inhibitor of metalloproteinase-2 through the activation of PI3K/Akt and ERK1/2. ${ }^{18,30}$ Moreover, a reduction in levels of myristic and lauric acids and an increase in the production of 20-HETE were observed. ${ }^{18}$ These alterations in levels of fatty acids were proposed before by Zollner et al, where CYP4Z1 metabolized myristic and lauric acids into different monohydroxylated metabolites and converted arachidonic acid to 20-HETE. ${ }^{18,20}$ However, the production of 20-HETE metabolites appears to contradict a recent study that indicates the absence of these metabolites in the arachidonic acid reaction with CYP4Z1 and generation of 14, 15-epoxyeicosatrienoic acid (14, 15-EET), a ligand confirmed to enhance tumour angiogenesis and growth. ${ }^{31}$ Additionally, CYP4Z1 was found to contribute to promoting the stemness and resistance of breast cancer cells to tamoxifen. ${ }^{30}$ All of these observations strongly support the hypothesis that CYP4Z1 may be associated with tumour metastasis.

Recent clinical studies indicating the potential roles of CYP4Z1 in tumour malignancy have stimulated interest in the biochemical properties of CYP4Z1. In this regard, a combined in vitro and silico framework has identified Arg487 as an important residue for substrate binding of CYP4Z1. ${ }^{22}$ The latest screening for substrate identification for CYP4Z1, expressed in permeabilized cells of fission yeast, revealed luciferin benzyl ether as the best luminogenic substrate. ${ }^{23}$ Recently, a selective mechanism-based inhibitor, 8-[(1 H-benzotriazol-1-yl)amino]octanoic acid, was developed, showing a potent inhibition of 14, 15EET production in breast cancer cells expressing CYP4Z1 as opposed to a low inhibitory profile against other CYPs. ${ }^{24}$ These recent efforts may open new pathways to the rational design of selective CYP4Z1 inhibitors and prodrugs.

\section{Conclusions}

The current study is the first reporting CYP4Z1 expression in relation to colon tissue types, including normal, benign, primary, and metastatic colon tissues. CYP4Z1 expression was increased in tumour tissues compared to normal tissues, as well as in metastatic tissues compared to benign and primary colon tumours. This unique expression was observed at a high rate in tumours of lymph node metastasis, high tumour histological grades, and late stages of the disease. Importantly, the differential in the expression of CYP4Z1 between colon tissue types may provide the basis for a risk stratification tool in colon cancers. Moreover, CYP4Z1 enzyme may be utilized as a biomarker or an actionable target for novel therapy development in colon cancer.

\section{Abbreviations}

CYP, cytochrome P450; CYP4Z1, cytochrome 4Z1;14,15EE,14, 15-epoxyeicosatrienoic acid; FFPE, formalin-fixed and paraffin-embedded; 20-HETE, 20-

hydroxyeicosatetraenoic acid.

\section{Acknowledgments}

The authors are very thankful to all the associated personnel in any reference who contributed towards the purpose of this research. Moreover, a special thanks to the Deanship of Scientific Research at Mutah University. 


\section{Disclosure}

The authors report no conflicts of interest in this work.

\section{References}

1. Bray F, Ferlay J, Soerjomataram I, Siegel RL, Torre LA, Jemal A. Global cancer statistics 2018: GLOBOCAN estimates of incidence and mortality worldwide for 36 cancers in 185 countries. CA Cancer J Clin. 2018;68(6):394-424.

2. Torre LA, Bray F, Siegel RL, Ferlay J, Lortet-Tieulent J, Jemal A. Global cancer statistics, 2012. CA Cancer J Clin. 2012;65(2):87-108. doi: $10.3322 /$ caac. 21262

3. Siegel RL, Miller KD, Jemal A. Cancer statistics, 2020. CA Cancer $J$ Clin. 2020;70(1):7-30. doi:10.3322/caac.21590

4. Kuipers EJ, Grady WM, Lieberman D, et al. Colorectal cancer. Nat Rev Dis Primers. 2015;1:15065. doi:10.1038/nrdp.2015.65

5. Al-Saraireh YM, Alshammari FO, Youssef AM, et al. Screening of glypican-6 expression in benign, primary and metastatic colon cancers. Clin Med Insights Oncol. 2021;15:11795549211036419. doi:10.1177/11795549211036419

6. Al-saraireh YM, Alshammari FOFO, Youssef AMM, et al. Profiling of CYP4Z1 and CYP1B1 expression in bladder cancers. Sci Rep. 2021;11(1):5581. doi:10.1038/s41598-021-85188-4

7. Murray GI, Patimalla S, Stewart KN, Miller ID, Heys SD. Profiling the expression of cytochrome P450 in breast cancer. Histopathology. 2010;57(2):202-211. doi:10.1111/j.1365-2559.2010.03606.x

8. Alshammari F, Al-Saraireh YM, Youssef AMM, Al-Sarayra YM, Alrawashdeh HM. Cytochrome P450 1B1 overexpression in cervical cancers: cross-sectional study. Interact J Med Res. 2021;10(4): e31150. doi: $10.2196 / 31150$

9. Edson KZ, Rettie AE. CYP4 enzymes as potential drug targets: focus on enzyme multiplicity, inducers and inhibitors, and therapeutic modulation of 20-hydroxyeicosatetraenoic acid (20-HETE) synthase and

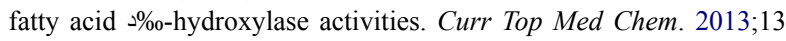
(12):1429-1440. doi:10.2174/15680266113139990110

10. Rieger MA, Ebner R, Bell DR, et al. Identification of a novel mammary-restricted cytochrome P450, CYP4Z1, with overexpression in breast carcinoma. Cancer Res. 2004;64(7):2357-2364. doi:10.1158/0008-5472.can-03-0849

11. Downie D, McFadyen MC, Rooney PH, et al. Profiling cytochrome P450 expression in ovarian cancer: identification of prognostic markers. Clin Cancer Res. 2005;11(20):7369-7375. doi:10.1158/ 1078-0432.CCR-05-0466

12. Tradonsky A, Rubin T, Beck R, Ring B, Seitz R, Mair S. A search for reliable molecular markers of prognosis in prostate cancer: a study of 240 cases. Am J Clin Pathol. 2012;137(6):918-930. doi:10.1309/ AJCPF3QWIG8FWXIH

13. Al-saraireh Yousef M, NS Alboaisa, Mohammad AH, Omar H, Sameeh A-S, Al-Shuneigat Jehad M, Nofal mohammad N. Screening of cytochrome $4 Z 1$ expression in human non-neoplastic, pre-neoplastic and neoplastic tissues. ecancer. 2020;14:1114.

14. Yang X, Hutter M, Goh WWB, Bureik M. CYP4Z1 - A human cytochrome p450 enzyme that might hold the key to curing breast cancer. Curr Pharm Des. 2017;23(14):2060-2064. doi:10.2174/ 1381612823666170207150156

15. Al-saraireh YM, Alshammari FO, Youssef AM, et al. Cytochrome $4 \mathrm{Z} 1$ expression is associated with unfavorable survival in triple-negative breast cancers. Breast Cancer. 2021;13:565-574.
16. Al-saraireh YM, Alshammari FOFO, Youssef AMM, et al. Cytochrome 4Z1 expression is correlated with poor prognosis in patients with cervical cancer. Curr Oncol. 2021;28(5):3573-3584. doi:10.3390/curroncol28050306

17. Khayeka-Wandabwa C, Ma X, Cao X, et al. Plasma membrane localization of CYP4Z1 and CYP19A1 and the detection of anti-CYP19A1 autoantibodies in humans. Int Immunopharmacol. 2019;73:64-71. doi:10.1016/j.intimp.2019.05.003

18. Yu W, Chai H, Li Y, et al. Increased expression of CYP4Z1 promotes tumor angiogenesis and growth in human breast cancer. Toxicol Appl Pharmacol. 2012;264(1):73-83. doi:10.1016/j.taap.2012.07.019

19. Savas U, Hsu MH, Griffin KJ, Bell DR, Johnson EF. Conditional regulation of the human CYP4X1 and CYP4Z1 genes. Arch Biochem Biophys. 2005;436(2):377-385. doi:10.1016/j.abb.2005.02.022

20. Zollner A, Dragan CA, Pistorius D, et al. Human CYP4Z1 catalyzes the in-chain hydroxylation of lauric acid and myristic acid. Biol Chem. 2009;390(4):313-317. doi:10.1515/BC.2009.030

21. McDonald MG, Ray S, Amorosi CJ, et al. Expression and functional characterization of breast cancer-associated cytochrome P450 4Z1 in saccharomyces cerevisiae. Drug Metab Dispos. 2019;45 (12):1364-1371. doi:10.1124/dmd.117.078188

22. Du W, Machalz D, Yan Q, Sorensen EJ, Wolber G, Bureik M. Importance of asparagine-381 and arginine-487 for substrate recognition in CYP4Z1. Biochem Pharmacol. 2020;174:113850. doi:10.1016/j.bcp.2020.113850

23. Yan Q, Machalz D, Zlllner A, Sorensen EJ, Wolber G, Bureik M. Efficient substrate screening and inhibitor testing of human CYP4Z1 using permeabilized recombinant fission yeast. Biochem Pharmacol. 2017;15(146):174-187. doi:10.1016/j.bcp.2017.09.011

24. Kowalski JP, McDonald MG, Pelletier RD, Hanenberg H, Wiek C, Rettie AE. Design and characterization of the first selective and potent mechanism-based inhibitor of cytochrome P450 4Z1. J Med Chem. 2020;63(9):4824-4836. doi:10.1021/acs.jmedchem.0c00101

25. Fleming M, Ravula S, Tatishchev SF, Wang HL. Colorectal carcinoma: pathologic aspects. J Gastrointest Oncol. 2012;3(3):153-173. doi:10.3978/j.issn.2078-6891.2012.030

26. Alnabulsi A, Swan R, Cash B, Murray GI. The differential expression of omega-3 and omega- 6 fatty acid metabolising enzymes in colorectal cancer and its prognostic significance. Br J Cancer. 2017;116 (12):1612-1620. doi:10.1038/bjc.2017.135

27. Kumarakulasingham M, Rooney PH, Dundas SR, et al. Cytochrome p450 profile of colorectal cancer: identification of markers of prognosis. Clin Cancer Res. 2005;11(10):3758-3765. doi:10.1158/ 1078-0432.CCR-04-1848

28. Murray GI, Patimalla S, Stewart KN, Miller ID, Heys SD. Profiling the expression of cytochrome P450 in breast cancer. Histopathology. 2009;57(2):202-211. doi:10.1111/j.1365-2559.2010.03606.x

29. Nunna V, Jalal N, Bureik M. Anti-CYP4Z1 autoantibodies detected in breast cancer patients. Cell Mol Immunol. 2017;14(6):572-574. doi:10.1038/cmi.2017.21

30. Zheng L, Guo Q, Xiang C, et al. Transcriptional factor six2 promotes the competitive endogenous RNA network between CYP4Z1 and pseudogene CYP4Z2P responsible for maintaining the stemness of breast cancer cells. J Hematol Oncol. 2019;12(1):23. doi:10.1186/ s13045-019-0697-6

31. McDonald MG, Ray S, Amorosi CJ, et al. Expression and functional characterization of breast cancer-associated cytochrome P450 4Z1 in Saccharomyces cerevisiae. Drug Metab Dispos. 2017;45 (12):1364-1371. doi:10.1124/dmd.117.078188 


\section{Publish your work in this journal}

OncoTargets and Therapy is an international, peer-reviewed, open access journal focusing on the pathological basis of all cancers, potential targets for therapy and treatment protocols employed to improve the management of cancer patients. The journal also focuses on the impact of management programs and new therapeutic agents and protocols on patient perspectives such as quality of life, adherence and satisfaction. The manuscript management system is completely online and includes a very quick and fair peer-review system, which is all easy to use. Visit http://www.dovepress.com/ testimonials.php to read real quotes from published authors.

Submit your manuscript here: https://www.dovepress.com/oncotargets-and-therapy-journal 